\title{
Influência do PH Inicial do Caldo de Cana-de-Açúcar na Produção de Levana por Zymomonas Mobilis ATCC 31821
}

\section{Influence of the initial ph of sugar cane juice on the production of levan by Zymomonas mobilis ATCC 31821.}

\author{
Marcia Sadae Tano ${ }^{1}$; João Batista Buzato ${ }^{2}$
}

\begin{abstract}
Resumo
Foi investigada a influência do $\mathrm{pH}$ inicial da garapa em alta concentração de açúcar para produção de levana por Zymomonas mobilis ATCC31821. Os pH iniciais dos meios foram 5,4; 5,9 e 6,3 e as concentrações de levana obtidas foram 1,66; 2,54 e 3,83 g/L, respectivamente, após 48 horas de cultivo. A concentração final de levana, em meio de $\mathrm{pH}$ inicial de 6,3 e 5,9 foi, respectivamente, $130 \%$ e $53 \%$ superior àquela obtida em meio de $\mathrm{pH}$ inicial de 5,4. O fator de produção em $\mathrm{pH}$ inicial 5,9 foi 87,.5\% e em $\mathrm{pH}$ 6,3 foi 162,5\% superiores em relação àquele obtido em $\mathrm{pH}$ inicial 5,4

Palavras-chave: Levana, pH, Zymomonas mobilis, caldo de cana-de-açúcar.
\end{abstract}

\section{Summary}

The influence of initial $\mathrm{pH}$ of sugar cane juice in high concentration was investigated for levan production by Zymomonas mobilis. In the initial $\mathrm{pH}$ of medium of 5.4; 5.9 and 6.3 the levan concentration achieved 1.66; 2.54 and $3.83 \mathrm{~g} / \mathrm{L}$, respectively, in 48 hours of fermentation. The final levan concentration in the initial $\mathrm{pH}$ of 6.3 and 5.9 increased 130 and $53 \%$ when compared to concentration at initial $\mathrm{pH} 5.4$ in the medium. The levan yield at $\mathrm{pH} 5.9$ was $87.5 \%$ and at $\mathrm{pH} 6.3$ was $162.5 \%$ superior in relation to that obtained at initial $\mathrm{pH} 5.4$.

Key Words: Levan, pH, Zymomonas mobilis, sugar cane juice.

\section{Introdução}

Geralmente, emprega-se a bactéria Z. mobilis para a produção de etanol a partir da sacarose, frutose e glicose (Falcão de Morais et al., 1993) . Porém, na fermentação, em alta concentração inicial de sacarose, a taxa de hidrólise deste dissacarídeo é maior do que o consumo dos seus monossacarídeos (PARKER et al., 1997; TANO et al., 2000). Conseqüentemente, os monossacarídeos acumulam-se no caldo de fermentação e favorecem a formação de subprodutos, como a levana e o sorbitol, o que re-

\footnotetext{
${ }^{1}$ Farmacêutica-Bioquímica. Pós-graduada em Microbiologia - Departamento de Microbiologia - UEL. Departamento de Bioquímica - Universidade Estadual de Londrina, Caixa Postal 6001, Londrina - PR, CEP 86051-900, Fone: (043) 371-4270, Fax (043) 371-4216.

${ }^{2}$ Docente do Departamento de Bioquímica da UEL
} 
duz o rendimento em etanol (KANNAN et al.,1997; PARKER et al., 1997)

Levana, um bioexopolímero de frutose, de alta massa molecular é formado pela atividade da enzima levansacarase e depende da disponibilidade de frutose acumulada no meio, após a hidrólise da sacarose (KANNAN et al., 1997; PARKER et al., 1997). Atribui-se a essa enzima uma dupla função, pois ela atua também na hidrólise da sacarose .

Levana é um produto de importância para as indústrias de alimentos como espessante e adoçante (Ananthalakshmy \& Gunasekaran.1999), farmacêutica (CALAZANS et al.,1997; FALCÃO DE MORAIS et al.,1993), cosmecêutica e de toileteria (SUTHERLAND, 1998). No entanto, a sua efetiva aplicação é dificultada pela sua baixa bioprodutividade (ANANTHALAKSMY; GUNASEKARAN, 1999; SUTHERLAND, 1998).

A influência da temperatura de cultivo sobre a composição do meio de fermentação e a concentração do extrato de levedura nesse meio foram estudadas como $\mathrm{Z}$ mobilis, com o objetivo de aumentar a produção de etanol e reduzir a formação de levana (ANANTHALAKSHMY; GUNASEKARAN, 1999; CRITTENDEN; DOELLE, 1994; DIEZ; YOKOYA,1996; FAVELLA TORRES; BARATTI, 1987).

São raros os trabalhos existentes que visam a melhorar o desempenho da bactéria para a produção de levana.

Por conseguinte, este trabalho teve a finalidade de avaliar a influência do $\mathrm{pH}$ inicial do caldo de cana-de-açúcar para a produção de levana por $Z$. mobilis ATCC 31821

\section{Materiais e métodos}

Microrganismo: Z. mobilis ATCC 31821.

Meio de manutenção: O meio foi preparado com 50 $\mathrm{g} / \mathrm{L}$ de sacarose e os outros componentes foram utilizados nas mesmas concentrações do meio de fermentação. Após incubação por 24 horas a $30^{\circ} \mathrm{C}$, as culturas foram mantidas a $4^{\circ} \mathrm{C}$ e reativadas a cada 30 dias.

Meio de fermentação (g/L): Açúcar redutor total (ART) do caldo de cana-de-açúcar 150; extrato de levedura 5; sulfato de magnésio hepta hidratado 0,5; sulfato de amônia 1; fosfato de potássio 1 . O volume de trabalho foi de $100 \mathrm{ml}$. Os valores de $\mathrm{pH}$ foram acertados com solução de KOH 50\% (pH 5,9; 6,3 ), a concentração celular inicial no meio de fermentação foi de $0,2 \mathrm{mg} / \mathrm{ml}$ e a fermentação foi conduzida a $28^{\circ} \mathrm{C}$ e $180 \mathrm{rpm}$. Os ensaios experimentais foram feitos com 2 repetições em triplicatas.

Métodos analíticos: A biomassa foi verificada por absortimetria em $605 \mathrm{~nm}$ contra água destilada. O correspondente peso seco foi obtido a partir de uma curva padrão estabelecida pela absorbância versus peso seco. A levana foi separada do caldo sobrenadante por precipitação pelo etanol a $75 \%$ e, após a hidrólise ácida a $65^{\circ} \mathrm{C}$ por 30 minutos, o polímero foi quantitifcado como como açúcar redutor (AR). As dosagens de AR e ART foram determinadas pelo Método de Somogy (1945), em $540 \mathrm{~nm}$. $\mathrm{O} \mathrm{pH}$ foi verificado por potenciometria (marca HANNA instruments modelo HI 9321). O etanol foi quantificado por cromatografia gasosa (marca SHIMADZU modelo CG-17A, coluna Dbwax e comprimento $30 \mathrm{~cm} / 0,25 \mathrm{~cm}$ na temperatura de $60^{\circ} \mathrm{C}$, injetor a $150^{\circ} \mathrm{C}$ e detector a $250^{\circ} \mathrm{C}$ ).

\section{Resultados e discussão}

A Tabela 1 mostra as produções de levana e os parâmetros cinéticos do consumo de açúcar obtido da fermentação do caldo de cana, em ART inicial (ARTi) de $150 \mathrm{~g} / \mathrm{L}$, conduzidos em 3 diferentes $\mathrm{pH}$ iniciais utilizando a Z. mobilis ATCC 31821. Os pH iniciais dos meios de fermentações estudados foram 5,$4 ; 5,9$ e 6,3 e os $\mathrm{pH}$ finais atingiram, seqüencialmente: 3,$82 ; 3,75$ e 3,88 , no final das fermentações (48 horas). 


\begin{tabular}{lcccc}
\hline \multicolumn{2}{c}{$\mathrm{pH}$ inicial } & 5,4 & 5,9 & 6,3 \\
\hline ART inicial & $\mathrm{g} / \mathrm{L}$ & 151,98 & 151,53 & 151,69 \\
ART cons. & $\mathrm{g} / \mathrm{L}$ & 68,97 & 55,86 & 60,66 \\
AR inicial & $\mathrm{g} / \mathrm{L}$ & 15,30 & 14,98 & 13,84 \\
AR residual & $\mathrm{g} / \mathrm{L}$ & 60,75 & 49,38 & 50,33 \\
Levana & $\mathrm{g} / \mathrm{L}$ & 1,66 & 2,54 & 3,83 \\
YL/S & $\mathrm{g} / \mathrm{g}$ & 0,024 & 0,045 & 0,063 \\
YP/S & $\mathrm{g} / \mathrm{g}$ & 0,38 & 0,37 & 0,34 \\
YX/S & $\mathrm{g} / \mathrm{g}$ & 0,034 & 0,028 & 0,032 \\
pH final & & 3,82 & 3,75 & 3,88
\end{tabular}

NOMENCLATURA: ART = açúcar redutor total; $\mathrm{AR}=$ açúcar redutor; YL/S = coeficiente de rendimento em levana ( $\mathrm{g}$ levana/ $\mathrm{g}$ açúcar consumido); YP/S = coeficiente de rendimento em etanol (g etanol/g açúcar consumido); YX/S = coeficiente de rendimento em biomassa ( $\mathrm{g}$ biomassa/g de açúcar consumido).

As bioproduções de levana nas fermentações do caldo de cana em pH 5,4; 5,9 e 6,3 foram 1,66; 2,54 e $3,83 \mathrm{~g} / \mathrm{L}$, respectivamente (Tabela 1). Comparativamente, as produções obtidas em levana em $\mathrm{pH} 5,9$ e 6,3 representam, respectivamente, um aumento em 53 e $130 \%$ com relação ao pH inicial 5,4 (referência $=100 \%$ ).

Outros autores, empregando diferentes cepas de Z. mobilis verificaram que a produção de levana foi bastante diversificada. Ananthalakshmy e Gunasekaran (1999) alcançaram valores de 12,6 g/ L com Z. mobilis B-4286 em 150g de sacarose/L, em 16 horas. Por outro lado, Yoshida et al. (1990) utilizaram a mesma concentração de sacarose e obtiveram produções de levana de $35 \mathrm{~g} / \mathrm{L}$ com $Z$. mobilis IN-17-10 e 3,1 g/L com Z. mobilis IFO 13757, em 3 dias, vale ressaltar que, nesses trabahos, o substrato acrescentado ao meio de fermentação foi a sacarose e não o caldo de cana in natura.

Os consumos de açúcar em pH 5,4; 5,9 e 6,3 foram 68,97; 55,86 e 60,66 g/L, respectivamente. Apesar da alta disponibilidade de açúcar (ARTi) no meio, observou-se um baixo consumo de açúcar e elevada quantidade de ARTr (residual), que é dada pela diferença do ARTi - ARTc (consumido), no final das fermentações. Esses consumos insatisfatórios de açucar podem ser resultantes da osmolaridade do meio e, também, da presença de inibidores no caldo de cana (JOACHIMSTHAL et al.,1998; PARK; BARATTI, 1993; TANO et al., 2000)

Paralelamente, as concentrações de ARr (residual) encontradas no final das fermentações foram 60,$75 ; 49,38$ e 50,33 g/L, respectivamente, demonstrando uma utilização incompleta de monossacarídeos, que se acumularam no caldo de fermentação (Tabela 1).

Rogers et al. (1982) observaram que aproximadamente $10 \%$ da sacarose (ARTc) foram convertidos para levana por Z. mobilis ATCC 31821. Em nossos resultados, nos cultivos em pH 5,4; 5,9 e 6,3 foram convertidos para levana, seqüencialmente, 2,$41 ; 4,55$ e $6,31 \%$ do açúcar consumido do caldo de cana. Assim, nossos valores obtidos foram inferiores ao mencionado por estes autores. Todavia, a melhor conversão do açúcar para levana foi obtido quando o $\mathrm{pH}$ inicial foi 6,3.

Os resultados deste trabalho mostraram-se mais compatíveis com o parâmetro citado por Viikari (1984) onde $2 \%$ da sacarose (ARTi) foi convertido para levana. Verificamos que, no meio de cultivo em pH 5,4; 5,9 e 6,3, o valor de açúcar inicial convertido em levana foi respectivamente: 1,09; 1,68 e 2,52\%. Portanto o aumento da conversão do açúcar para levana acompanhou a elevação dos $\mathrm{pH}$ iniciais.

Os rendimentos em levana (YL/S) aqui obtidos em $\mathrm{pH}$ 5,4; 5,9 e 6,3 foram: 0,024; 0,045 e 0,063, respectivamente. Proporcionalmente, estes rendimentos foram 87,5 e $162,5 \%$ superiores aos obtidos em pH 5,4.

Os rendimentos em etanol (YP/S) em pH 5,4; 5,9 e 6,3 foram, respectivamente, 0,$38 ; 0,37$ e 0,34 . Os valores de rendimento em $\mathrm{pH}$ 5,4 e 5,9 não apresentam alteração significativa. No entanto, em pH 6.3 o rendimento obtido de levana acarretou uma diminuição do rendimento em etanol (Tabela 1). Esses resultados estão de acordo com Diez e Yokoya (1996) e Viikari (1984) 
Por outro lado, o rendimento em biomassa (YX/ S) foi maior em pH 5,4 do que em pH 5,9 e 6,3 (Tabela 1). Os rendimentos em $\mathrm{pH} 5,4 ; 5,9$ e 6,3 foram: 0,034; 0,028 e 0,032 respectivamente, que são compatíveis com os resultados obtidos por Diez e Yokoya (1996).

Vale destacar que, segundo os estudos de Lyness e Doelle (1983), que purificaram a enzima levansacarase de uma cultura de Z. mobilis NCIB 11199, a atividade enzimática para hidrólise da sacarose foi melhor em pH 6,5. Crittenden e Doelle (1994) estudaram a atividade levansacarase da $Z$. mobilis UQM 2716 e concluíram que para a formação de levana o pH 5,5 foi melhor. Posteriormente, Ananthalakshmy e Gunasekaran (1999) obtiveram uma maior produção de levana em $\mathrm{pH}$ inicial 5,0 do que em pH 6,0 e 7,0. Em somatória, esses autores observaram em $Z$. mobilis a existência de uma atividade descrita como levanase.

Diez e Yokoya (1996), por sua vez, demonstraram a influência do $\mathrm{pH}$ inicial do meio de fermentação, em cultura contínua, com Z. mobilis ATCC 31821. Na fermentação com pH inicial em 5,7, mantido sob controle, a produção de levana foi de 2,86 $\mathrm{g} / \mathrm{L}$, porém quando este $\mathrm{pH}$ inicial não esteve sob controle a produção foi de $3,77 \mathrm{~g} / \mathrm{L}$.

Muitas são as variáveis interferentes para a produção de levana. Com base nos resultados deste trabalho, podemos concluir que na, fermentação do caldo de cana em alta concentração de açúcar inicial com Z. mobilis ATCC 31821, a produção de levana foi superior quando o $\mathrm{pH}$ inicial foi 6,3 em relação aos $\mathrm{pH}$ iniciais 5,4 e 5,9.

Os resultados indicam que Z. mobilis parece dispor de mecanismos bioquímicos reguladores da produção de levana, como substância de reserva temporária, quando há alta disponibilidade de frutose no caldo de fermentação. Para isso, ele pode utilizar-se da atividade levanase, como afirmaram Ananthalakshmy e Gunasekaran (1999).

\section{Referências}

ANANTHALAKSHMY, V.K.; GUNASEKARAN, P. Optimization of levan production by Zymomonas mobilis. Brazilian Archives Biology Technology, 42, n.3, p.292297, 1999.

CALAZANS, G.M.T. et al. Antitumor activity of levans produced by Zymomonas mobilis strains. Biotechnology Letters, v.19, p.19-21, 1997.

CRITTENDEN, R.G.; DOELLE, H.W. Identification and characterisation of the extracellular sucrases of Zymomonas mobilis UQM 2716 (ATCC 39676). Applied Microbiology Biotechnology, v.41, n.3, p.302-308, 1994.

DIEZ, J.C.; YOKOYA, F. Efeito da temperatura e $\mathrm{pH}$ na produção de etanol e levana durante a fermentação de sacarose por Zymomonas mobilis. Arquivos Biologia Tecnologia, v.39, n.1, p.129-137, 1996.

FALCÃO DE MORAIS, J.O. et al. Zymomonas mobilis research in the Pernambuco Federal University. Journal Biotechnology, v.31, p.75-91, 1993.

FAVELA TORRES, E.; BARATTI, J. The effect of pH, temperature and sucrose concentration on high productivity continuous ethanol fermentation using Zymomonas mobilis. Applied Microbiology Biotechnology, v.27, p.121-128, 1987.

JOACHIMSTHAL, E. et al. A mutant of Zymomonas mobilis ZM4 capable of ethanol production from glucose in the presence of high acetate concentrations. Biotechnology Letters, v.20, n.2, p.137-142, 1998.

KANNAN, T.R.; SANGILIYANDI, G.; GUNASEKARAN, P. Influence of intra- and extracellular sucrases of Zymomonas mobilis on the ethanol production and by-product formation. Biotechnology Letters, v.19, n.7, p.661-664, 1997.

LYNESS, E.W.; DOELLE, H.W. Levansucrase from Zymomonas mobilis. Biotechnology Letters, v.5, n.5, p.345-350, 1983.

PARKER, C. et al. Kinetics of sugar transport and phosphorylation influence glucose and fructose cometabolism by Zymomonas mobilis. Applied Environmental Microbiology, v.63, n.9, p.3519-3525, 1997.

PARK, S.C.; BARATTI, J. Effects of potassium chloride on ethanol production by an osmotolerant mutant of Zymomonas mobilis. Applied Microbiology Biotechnology, v.38, p.542-549, 1993.

ROGERS, P.L. et al. Ethanol production by Zymomonas mobilis. Advance Biochemistry Engineering, v.23, p.3784, 1982. 
Influência do PH Inicial do Caldo de Cana-de-Açúcar na Produção de Levana por Zymomonas Mobilis Atcc 31821

SOMOGYI, M.A. A new reagent for the determination of sugar. Biol. Chemistry, v.160, p.61-68, 1945.

SUTHERLAND, I.W. Novel and established applications of microbial polysaccharides. TIBTECH, v.16, p.41-46, 1998.

TANO, M.S.; BUZATO, J.B.; CELliGOI, M.A.P.C. Sugar cane juice fermentation by Zymomonas mobilis CP4 subjected to inhibition by ethanol and high initial concentration of substrate. Brazilian Archives Biology Technology, v.43, n.4, p.425-430, 2000.
VIIKARI, L. Formation of levan and sorbitol from sucrose by Zymomonas mobilis. Applied Microbiology Biotechnology, v.19, p.252-255, 1984.

YOSHIDA, Y., SUZUKI, R.; YAGI, Y. Production of levan by a Zymomonas sp. Journal Fermentation Bioengineering, v.70, n.4, p.269-271, 1990. 
